08

\title{
Модификация поверхности пленки иодида серебра при электронном облучении
}

\author{
(C) E.C. Бочкарева, ${ }^{1}$ А.И. Сидоров, ${ }^{1, \uparrow}$ А.В. Нащекин ${ }^{2}$ \\ ${ }^{1}$ Университет ИТМО, \\ 197101 Санкт-Петербург, Россия \\ ${ }^{2}$ Физико-технический институт им. А.Ф. Иоффре РАН, \\ 194021 Санкт-Петербург, Россия \\ ฯ e-mail: sidorov@oi.ifmo.ru
}

(Поступило в Редакцию 19 октября 2017 г.)

\begin{abstract}
Экспериментально показано, что облучение электронами поликристаллических пленок AgI приводит к формированию на их поверхности монослоя наночастиц серебра. Увеличение продолжительности облучения приводит к увеличению размера наночастиц, при этом их концентрация сначала увеличивается, а затем уменьшается. Рассмотрены механизмы образования наночастиц и динамики их роста. Показано, что основную роль в наблюдаемых эффектах играет процесс перекристаллизации Оствальда, а также процессы полевой миграции и изменение заряда наночастиц при облучении электронами относительно низкой энергии.
\end{abstract}

DOI: $10.21883 /$ JTF.2018.07.46179.2522

\section{Введение}

Развитие физики, химии и технологий требует создания новых перспективных функциональных материалов. Иодид серебра (AgI) известен как суперионный проводник, и его свойства исследуются на протяжении десятилетий. Нанокомпозиты, содержащие AgI, находят применение в фотографии, оптоэлектронике, фотонике, сенсорике и в фотокаталитических реакциях. Интересными свойствами обладают гибридные наноструктуры, состоящие из наночастиц (НЧ) $\mathrm{AgI}$ и $\mathrm{HЧ} \mathrm{Ag}$. Экситонная полоса поглощения $\mathrm{AgI}(\lambda=420-430 \mathrm{~nm})$ практически точно совпадает со спектральным положением плазмонной полосы поглощения $\mathrm{HЧ} \mathrm{Ag}(\lambda=390-420 \mathrm{~nm})$. Как показано в [1], это может приводить к возникновению резонанса Фано в таких гибридных наноструктурах. Наночастицы $\mathrm{Ag}$ благодаря плазмонному резонансу усиливают фотокаталитические свойства AgI [2-6].

Как правило, гибридные наноструктуры AgI-Ag синтезируют путем частичного фотолиза пленок или НЧ $\mathrm{AgI}$ УФ-излучением. Однако для ряда практических применений требуется создание наноструктур в нано- и микромасштабных областях. Например, в нано- и микрофлюидных устройствах $[7,8]$ необходимо проводить анализ микрообъемов вещества, проводить химические или фотокаталитические реакции в микро- и нанореакторах. В таких случаях наиболее удобным инструментом для модификации свойств пленок AgI является сфокусированный электронный луч. Электронный луч может быть сфокусирован в пятно диаметром менее $10 \mathrm{~nm}$. Локальное облучение электронами стекол, содержащих ионы металла, позволяет создавать НЧ металлов, таких как $\mathrm{Ag}, \mathrm{Cu}, \mathrm{Na}$ и др. как в приповерхностных слоях стекол, так и на их поверхности [9-12]. Поэтому в настоящей работе была поставлена задача исследования процессов, происходящих на поверхности поликристаллических пленок AgI при их локальном облучении электронами.

\section{Методика экспериментов}

В экспериментах использовались поликристаллические пленки AgI со средней толщиной $200 \mathrm{~nm}$ на полированных подложках из силикатного стекла. Для изготовления пленок AgI на подложки методом вакуумного напыления наносился слой $\mathrm{Ag}$ толщиной $100 \mathrm{~nm}$, после чего образцы обрабатывались в парах иода при комнатной температуре в течение $2 \mathrm{~h}$.

Для облучения электронами и получения изображений поверхности пленок использовался сканирующий электронный микроскоп (SEM) JSM-7001F (JEOL). Диаметр пятна в фокусе электронного луча был приблизительно равен $15 \mathrm{~nm}$. Использовались электроны с энергией $5 \mathrm{keV}$, электронный ток в пучке был равен $15 \mathrm{pA}$. Продолжительность облучения в каждом цикле составляла $20 \mathrm{~s}$. Доза облучения при этом была равна $8 \mathrm{mC} / \mathrm{cm}^{2}$. После каждого цикла облучения регистрировалось SEM-изображение. Общая продолжительность облучения составляла $140 \mathrm{~s}$. Облучение проводилось путем сканирования электронным лучом. Облученный участок имел размеры $2.2 \times 1.65 \mu \mathrm{m}$. Спектры оптической плотности измерялись с помощью волоконного спектрометра EPP2000-UVN-SR (StellarNet) при комнатной температуре.

\section{Экспериментальные результаты и обсуждение}

На рис. 1 показаны спектры оптической плотности пленки AgI до и после электронного облучения. Из 


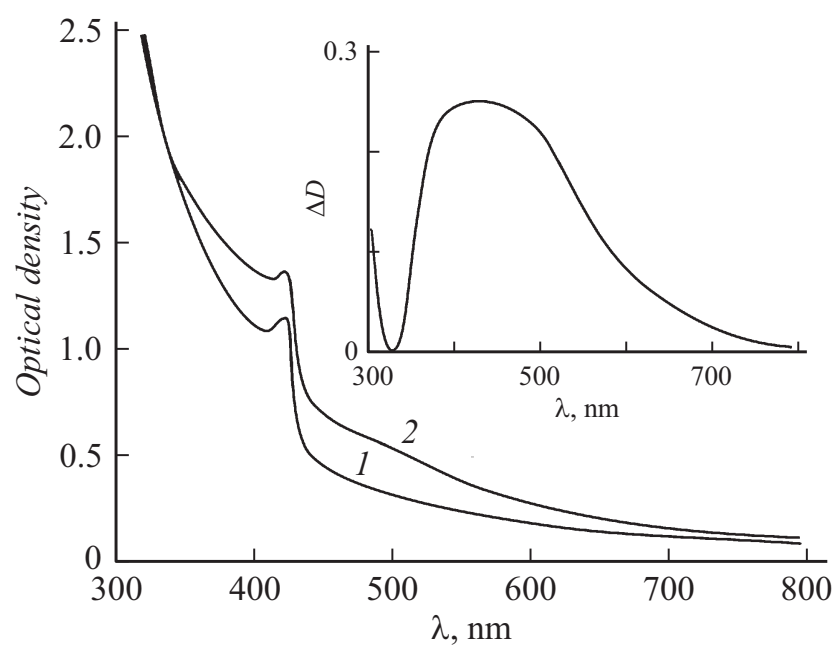

Рис. 1. Спектры оптической плотности пленки $\mathrm{AgI}$ до (1) и после (2) электронного облучения. На вставке - разностный спектр оптической плотности: $\Delta D=D_{2}-D_{1}$.

рисунка видно, что на обеих кривых присутствует полоса поглощения на $\lambda=420 \mathrm{~nm}$, типичная для экситонного поглощения кристаллического AgI. Электронное облуче- ние приводит к увеличению поглощения в спектральном интервале 350-650 nm. Разностный спектр оптической плотности (вставка на рис. 1) показывает появление в данном спектральном интервале новой полосы поглощения, характерной для плазмонного резонанса НЧ $\mathrm{Ag}$ [13]. Полоса поглощения имеет бо́льшую спектральную ширину, она асимметрична. Ширина и форма плазмонной полосы поглощения указывает на то, что она является суперпозицией плазмонных полос поглощения НЧ $\mathrm{Ag}$, имеющих большой разброс по форме и размерам [13].

SEM-изображения пленки AgI до и после облучения электронами показаны на рис. 2. Из рисунка видно, что пленка состоит из кристаллитов, размер которых варьирует от $100 \mathrm{~nm}$ до $3 \mu \mathrm{m}$. Пленка покрывает подложку неравномерно - имеются области, где кристаллиты отсутствуют (черные области на вставке в рис. 2,a). Кристаллиты имеют дефекты, возможно, в виде дислокаций (темные полосы на рис. 2). Облучение электронами приводит к появлению на поверхности кристаллитов монослоя НЧ Ag уже после 20 s облучения. Однако НЧ $\mathrm{Ag}$ формируются на поверхности кристаллитов неравномерно, но в первую очередь на границах кристаллитов и в области дефектов. Увеличение продолжительности
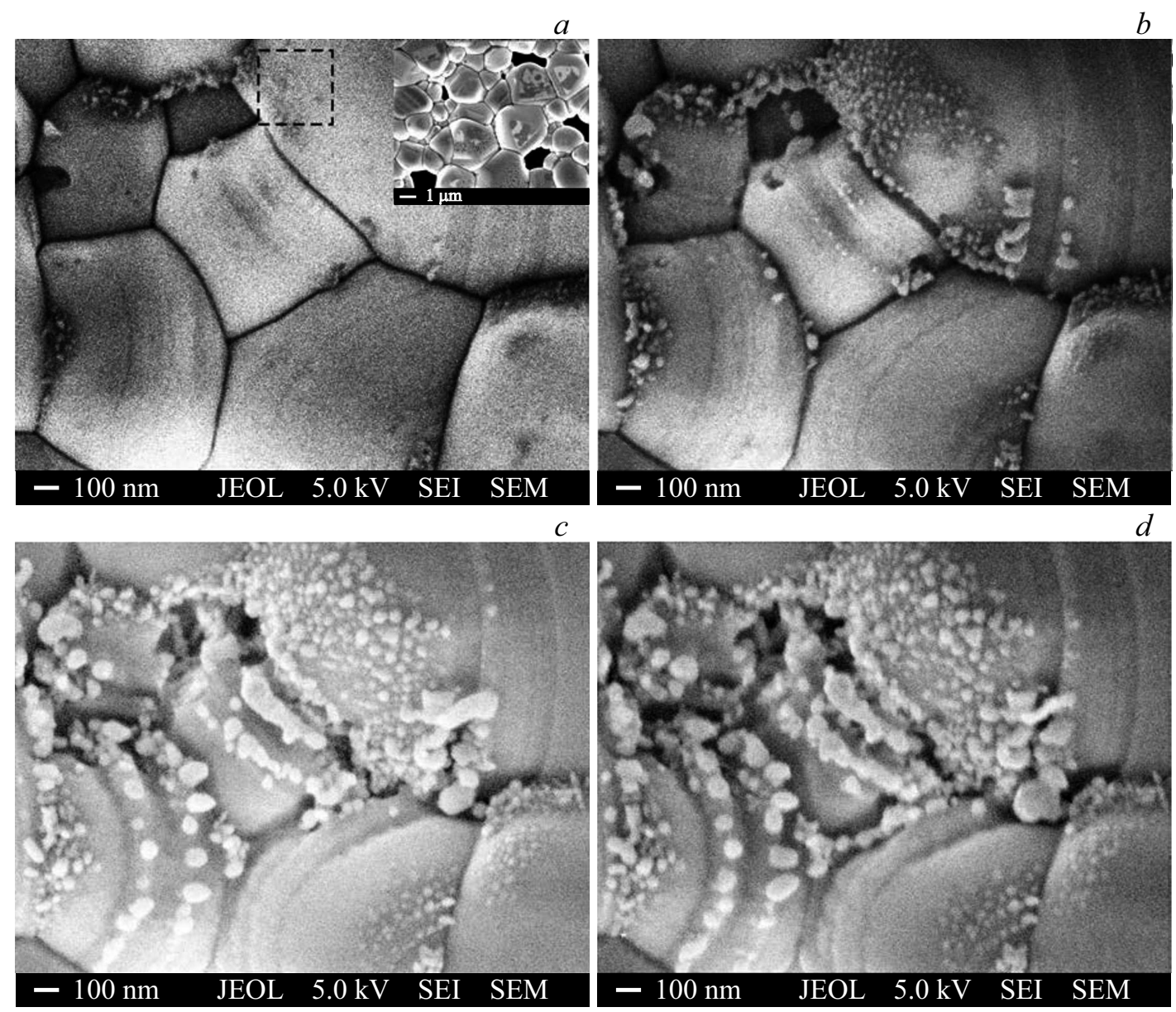

Рис. 2. SEM-изображения пленки AgI до и после облучения электронами. Продолжительность облучения: $a-20, b-60$, $c-120, d-140 \mathrm{~s}$. Масштаб $100 \mathrm{~nm}$. На вставке $(a)-$ SEM-изображение пленки до облучения электронами. Масштаб $1 \mu \mathrm{m}$. Квадрат в $(a)$, изображенный штриховой линией - область анализа концентрации и размеров НЧ Ag. 


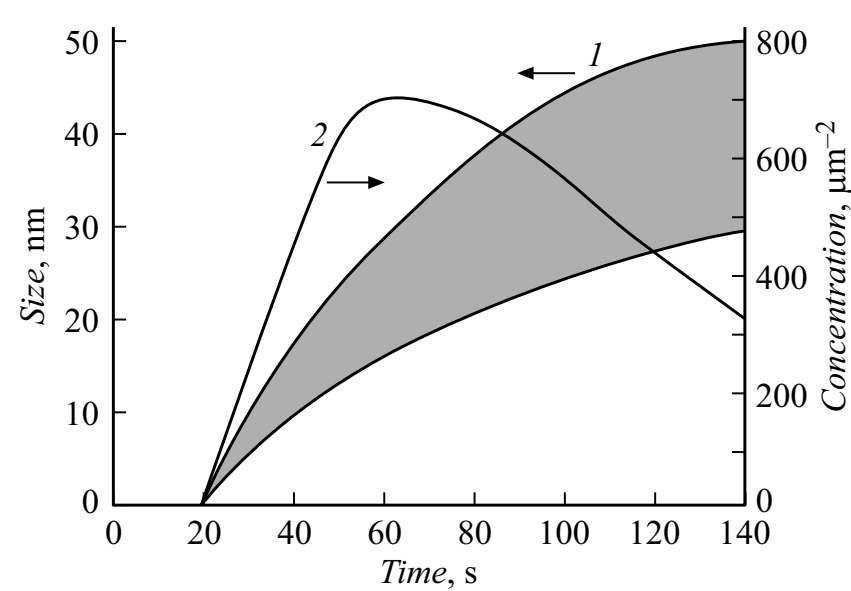

Рис. 3. Динамика роста НЧ Ag при электронном облучении пленки AgI: 1 - зависимость размера НЧ от продолжительности облучения, 2 - зависимость концентрации НЧ от продолжительности облучения.

облучения приводит к увеличению размеров НЧ $\mathrm{Ag}$ и на начальном этапе облучения к увеличению их концентрации. Причем на границах кристаллитов и в области дефектов увеличение размеров НЧ происходит значительно быстрее, чем в других областях кристаллитов. НЧ Ag имеют неправильную форму, а их максимальный размер достигает $100 \mathrm{~nm}$.

Выход серебра из пленки AgI на ее поверхность может быть объяснен следующими процессами. Расчет показывает, что электроны с энергией $5 \mathrm{keV}$ теряют свою энергию в слое $\mathrm{AgI}$ толщиной менее $100 \mathrm{~nm}$. При этом в приповерхностном слое AgI формируется область отрицательного заряда, поле которого вытягивает положительные ионы серебра из объема пленки. Термализованные электроны в данной области восстанавливают ионы серебра до нейтрального состояния, после чего атомы серебра получают возможность выйти на поверхность пленки и сформировать НЧ. Наличие дефектов облегчает перемещение ионов и атомов серебра по кристаллиту, поэтому НЧ Ag формируются в первую очередь в областях с высокой концентрацией дефектов.

Так как НЧ распределены по кристаллитам неравномерно, то для дальнейшего анализа динамики их роста была выбрана область кристаллита размером $300 \times 300 \mathrm{~nm}$, отмеченная на рис. 2, $a$ штриховой линией. Из рис. 2, $a$ видно, что концентрация дефектов в данной области незначительна. На рис. 3 показана динамика роста НЧ Ag при электронном облучении пленки AgI. Серая область на рисунке обозначает разброс НЧ по размерам. Из рисунка видно, что при увеличении продолжительности электронного облучения размер НЧ $\mathrm{Ag}$ увеличивается (кривая 1 на рис. 3). Максимальный размер НЧ после $140 \mathrm{~s}$ облучения составляет $50 \mathrm{~nm}$. На начальном этапе облучения концентрация НЧ увеличивается (кривая 2 на рис. 3 ), однако при продолжи- тельности облучения более $60 \mathrm{~s}$ начинается уменьшение концентрации НЧ.

Наблюдаемые эффекты могут быть объяснены с позиций теории перекристаллизации Оствальда (Ostwald's ripening) [14]. Согласно данной теории, с энергетической точки зрения более выгодным оказывается рост более крупных частиц. При этом мелкие частицы отдают свой материал более крупным, сливаются с ними и постепенно исчезают. В результате происходит увеличение размеров частиц и уменьшение их концентрации. В случае облучения НЧ $\mathrm{Ag}$ электронами относительно низких энергий появляется дополнительный механизм, вносящий вклад в динамику роста НЧ. Данные механизмы подробно рассмотрены в работе [15], в которой аналогичные эффекты наблюдались при облучении электронами НЧ Ag на поверхности стекла. Как показано в [15], более крупные НЧ Ag с большей вероятностью захватывают электроны с энергией $5 \mathrm{keV}$, приобретая при этом отрицательный заряд. Эффекты, связанные с вторичной электронной эмиссией, приводят к тому, что НЧ малого размера с большей вероятностью приобретают положительный заряд. Электрическое поле высокой напряженности, возникающее при этом, приводит к полевой ионизации ионов серебра с таких НЧ, которые могут быть захвачены отрицательно заряженными крупными НЧ. Кроме того, возникновение локальных электрических полей разного знака может приводить к полевой миграции положительно заряженных малых НЧ и к их слиянию с отрицательно заряженными крупными НЧ.

\section{Заключение}

Облучение электронами поликристаллических пленок AgI сопровождается формированием на их поверхности НЧ Ag. Увеличение продолжительности облучения приводит к увеличению размера НЧ, но их концентрация при этом сначала увеличивается, а затем уменьшается. При максимальной продолжительности облучения (140s) размер НЧ варьирует от 30 до $50 \mathrm{~nm}$. Концентрация НЧ достигает максимума при продолжительности облучения $60 \mathrm{~s}$ и составляет $700 \mu \mathrm{m}^{-2}$. Основную роль в наблюдаемых эффектах играет процесс перекристаллизации Оствальда, а также процессы полевой миграции и изменение заряда наночастиц при облучении электронами относительно низкой энергии.

Настоящая работа проведена при финансовой поддержке Министерства образования и науки Российской Федерации (проект 16.1651.2017/4.6). Электронномикроскопические исследования выполнены на оборудовании Федерального ЦКП „Материаловедение и диагностика в передовых технологиях“ при ФТИ им. А.Ф. Иоффе, поддержанного Минобрнауки России (уникальный идентификатор проекта RFMEFI62117X0018). 


\section{Список литературы}

[1] Андреева О.В., Сидоров А.И., Стаселько Д.И., Хрущева T.A. // ФТТ. 2012. Т. 54. С. 1215-1219. [Andreeva O.V., Sidorov A.I., Stasel'ko D.I., Khrushcheva T.A. // Phys. Sol. State. 2012. Vol. 54. P. 1293-1297.]

[2] Awazu K., Fujimaki M., Rockstuhl C., Tominaga J., Murakami H., Ohki Y., Yoshida N., Watanabe T. // J. Am. Chem. Soc. 2008. Vol. 130. P. 1676-1680.

[3] Bi Y.P., Ye J.H. // Chem. Commun. 2009. N 43. P. 6551-6553.

[4] Cao J., Luo B., Lin H., Chen S. // J. Mol. Catal. A. Chem. 2011. Vol. 344. P. 138-144.

[5] Yi Z.G., Ye J.H., Kikugawa N., Kako T., Ouyang S.X., StuartWilliams H., Yang H., Cao J.Y., Luo W.J., Li Z.S., Liu Y., Withers R.L. // Nature Mater. 2010. Vol. 9. P. 559-564.

[6] Jiang J., Zhang L.Z. // Chem.A. Europ. J. 2011. Vol. 17. P. 3710-3717.

[7] Zhang D., Men L., Chen Q. // Sensors. 2011. Vol. 11. P. 5360-5382. DOI: $10.3390 / \mathrm{s} 110505360$

[8] Hamish C., Hunt J., James S. // Microfluid. Nanofluid. 2008. Vol. 4. P. 53-79. DOI: 10.1007/s10404-007-0223-y

[9] Игнатьев А.И., Нащекин А.В., Неведомский В.М., Подсвиров О.А., Сидоров А.И., Соловьев А.П., Усов О.А. // ЖТФ. 2011. Т. 81. Вып. 5. С. 75-80. [Ignatiev A.I., Naschekin A.V., Nevedomsky D.M., Podsvirov O.A., Sidorov A.I., Soloviev A.P., Usov O.A. // Techn. Phys. Vol. 56. P. 662-667.]

[10] Подсвиров О.А., Сидоров А.И., Цехомский В.А., Востоков A.B. // ФТТ. 2010. Т. 52. С. 1776-1779. [Podsvirov O.A., Sidorov A.I., Tsikhomsky V.A., Vostokov A.V. // Phys. Sol. Stat. 2010. Vol. 52. P. 1906-1909.]

[11] Брунов В.С., Подсвиров О.А., Сидоров А.И., Чураев Д.В. // ЖТФ. 2014. Т. 84. Вып. 8. С. 112-117. [Brunov V.S., Podsvirov O.A., Sidorov A.I., Churaev D.V. // Techn. Phys. Vol. 59. P. 1215-1219.]

[12] Bochkareva E.S., Nikonorov N.V., Podsvirov O.A., Prosnikov M.A., Sidorov A.I. // Plasmonics. 2016. Vol. 11. N 1. P. 241-246.]

[13] Климов В.В. Наноплазмоника. М.: Физматлит, 2009. 480 с. [Klimov V.V. Nanoplasmonics. Pan Stanford, Singapore, 2014.]

[14] Ostwald $W$. // Zeitschriftfür Physika Lische Chemie. 1897. Vol. 22. P. 289-330.

[15] Сидоров А.И., Просников М.А., Боричева И.К. // ЖТФ. 2015. Т. 85. Вып. 12. С. 138-142. [Sidorov A.I., Prosnikov M.A., Boricheva B.K. // Thechn. Phys. 2015. Vol. 60. P. 1872-1876.] 